\title{
EL ENFOQUE ECOSISTEMICO EN EL MANEJO FORESTAL EN CENTROAMERICA
}

Campos, J., Villalobos, R. y Louman, B. Catedra Latinoamericana de Manejo Diversificado de Bosques Tropicales, Centro Agronómico Tropical de Investigación y Enseñanza (CATIE), jcampos@catie.ac.cr, www.catie.ac.cr

\section{RESUMEN}

El enfoque ecosistémico (EE) es un concepto relativamente nuevo en América Central, sin embargo muchos de sus principios han sido ya considerados en numerosas iniciativas de desarrollo forestal sostenible (MFS) en la Región. El desarrollo e implementación del EE en MFS en el corto y mediano plazo enfrenta muchos desafios, como un alto crecimiento de la población, fuertes tasas de deforestación, economías pequeñas y estancadas, factores que se combinan con pobreza rural y debilidad institucional. Esto debiera justificar que se de una mayor atención a los aspectos sociales, económicos e institucionales relacionados con EE en MFS en la Región.

Los procesos de cambios legislativos que han promovido descentralización del manejo forestal, a través de concesiones forestales comunitarias en Guatemala y mediante los gobiernos locales en Honduras y Nicaragua son buenos ejemplos de herramientas de conducción de EE - MFS. Otra herramienta importante para progresar hacia el EE - MFS en esta región ha sido el uso de mecanismos financieros, particularmente en Costa Rica y Guatemala, mereciendo especial atención el desarrollo de instrumentos de mercado (Ej. Pago por servicios ambientales) que proveen una compensación financiera por los servicios ambientales que los ecosistemas forestales pueden proporcionar a la comunidad. Importantes desarrollos se han producido también respecto del establecimiento de instituciones que promueven la integración regional (entre paises) y la implementación de aproximaciones ecoregionales, en ambos casos reconociendo que los esfuerzos de conservación requieren de más largas escalas espaciales y temporales y enfoques más integrados para el manejo de los recursos naturales (Ej. El corredor biológico mesoamericano, con sus numerosas iniciativas a diferentes escalas nacionales y locales).

Al nivel de la unidad de manejo forestal se han logrado avances de importancia en materia de desarrollo de tecnologias, legislación y mecanismos de mercado, como la certificación forestal que ha permitido una mayor protección de la estructura y funciones de los bosques mediante la implementación creciente de buenas prácticas forestales. Sin embargo, los reforzamientos legales y la promoción de incentivos para reducir cortas y comercializaciones ilegales de productos forestales, continúan siendo una prioridad de acción.

Los procesos crecientes de fragmentación de bosques, la pequeña escala de la unidades de manejo forestal y la importancia de de la interacción múltiple en el nivel de paisaje, lleva a sugerir que la promoción de EE - MFS debiera alcanzar mejores resultados si es articulado mediante iniciativas actuales y futuras de planificación de uso de la tierra y de manejo que tengan un enfoque más amplio e integrado, como las reservas de la biosfera, el manejo intergrado de cuencas, los bosques modelos y los corredores biológicos. 
Se requiere de mayores esfuerzos en I \& D para enfrentar la complejidad de usos y dudas que afectan el EE - MFS, para construir capacidad y ampliar la comunicación con los diversos grupos de interés, incluidos quienes hacen las politicas. Además, el apoyo al fortalecimiento de las organizaciones de productores y sus capacidades de emprender, la promoción de alianzas de aprendizaje y esfuerzos orientados al escalamiento de experiencias en iniciativas seleccionadas son la clave para el éxito del EE - MFS en América Central.

\section{SUMMARY}

The ecosystem approach (EA) is a relatively new concept in Central America; however, many of its principles have already been taken into account in many sustainable forest management (SFM) initiatives in this Region. Further development and implementation of an EA in SFM in the short and medium term faces many challenges, including high population growth, high deforestation rates, small and stagnant economies, combined with rural poverty and weak institutions. This might justify that greater attention should be given to social, economic and institutional issues related to EA-SFM in this region.

The processes of changes in legislation that promoted decentralization of forest management through community forest concessions in Guatemala and through local governments in Honduras and Nicaragua are good examples of drivers of the EA-SFM. Another important driver for the progress towards the EA-SFM in this region has been the use of financial mechanisms, particularly in Costa Rica and Guatemala, deserving especial attention the development of market instruments (e.g. payment for environmental services) that provide a financial compensation for the environmental services that forest ecosystems provide to society. Important developments have also taken place regarding the establishment of institutions promoting regional integration (among countries) and the implementation of ecoregional approaches, both acknowledging that conservation efforts require larger spatial and temporal scales and more integrated approaches to the management of natural resources (e.g. the Mesoamerican Biological Corridor with its many initiatives at different national and local scales).

At the forest management unit level there have been important advances regarding the development of technologies, legislation and market mechanisms such as forest certification, that have resulted in increased protection of forest structure and functions, through an increasing implementation of good forest practices. However, law enforcement and further promotion of incentives to reduce illegal logging and trade of forest products continue to be a priority for action.

The increasing process of forest fragmentation, the small size of the forest management units and the relevance of the multiple interactions at the landscape level, lead us to suggest that the promotion of EA-SFM might achieve better results if it is articulated within current and future land use planning and management initiatives that have a broader and more integrated scope, such as biosphere reserves, integrated watershed management, model forests and biological corridors.

More efforts are required in R\&D to address the complexity of issues and uncertainties affecting EA-SFM, to build capacity and enhance communication with the diversity of 
stakeholders, including policy-makers. Furthermore, support for strengthening organizations of producers and their entrepreneurial capacity, the promotion of learning alliances and further collaborative efforts aiming at scaling-up and scaling-out experiences in selected pilot initiatives are key for EA-SFM to succeed in Central America.

\section{INTRODUCCION}

A partir de Río 92, diversidad de convenciones y foros intergubernamentales, han generado nuevos paradigmas en el sector forestal que inciden gradualmente en las tendencias de manejo y las políticas relativas a los recursos forestales: Uno de los foros más relevantes es la Convención sobre la Diversidad Biológica (CDB), la cual propone el Enfoque Ecosistémico (EE) como marco de referencia para lograr sus objetivos. Este es un concepto relativamente nuevo en América Central y desconocido para la mayoría de los actores, sin embargo muchos de los elementos que se contemplan en sus principios ya se manifiestan en las iniciativas de manejo forestal sostenible (MFS) en esta Región, en particular durante la última década.

Los principios del enfoque ecosistémico, en su versión más resumida son:

1. La elección de los objetivos de la gestión de los recursos de la tierra, el agua y los recursos vivientes debe quedar en manos de la sociedad.

2. La gestión debe estar descentralizada hasta el nivel apropiado más bajo.

3. Quienes manejan el ecosistema deben tener en cuenta los efectos (reales o posibles) de sus actividades en los ecosistemas adyacentes.

4. Dados los posibles beneficios derivados de su gestión, es necesario comprender y gestionar el ecosistema en un contexto económico.

5. La conservación de la estructura y el funcionamiento de los ecosistemas debe ser prioritaria.

6. Los ecosistemas deben ser manejados dentro de los límites de su funcionamiento.

7. El enfoque ecosistémico debe ser aplicado a las escalas espaciales y temporales apropiadas.

8. Dadas las diversas escalas temporales y los efectos retardados que caracterizan los procesos ecosistémicos, los objetivos de manejo deberían establecerse para el largo plazo.

9. En la gestión debe reconocerse que el cambio es inevitable. 
10. En el enfoque debe procurar el equilibrio apropiado entre la conservación y la utilización de la diversidad biológica y su integración.

11. Deben tomarse en cuenta todas las formas de información pertinente, incluido el conocimiento científico, indigena, local, las innovaciones y las prácticas.

12. En el enfoque ecosistémico deben intervenir todos los sectores de la sociedad y las disciplinas cientificas pertinentes.

El grado de aplicación de los principios del EE sin embargo, es afectado por condiciones prevalecientes en la Región, entre ellas la alta tasa de deforestación y de degradación de ecosistemas forestales, que se estimó en 341 mil hectáreas anuales (1,6\%) para la década 1990-2000 y ha resultado en una cobertura forestal de apenas 34,9\% (FAO, 2002).

Entre las causas de esta degradación están las politicas que han favorecido el desarrollo agropecuario y la colonización, antes que el manejo de los ecosistemas forestales (Campos et al., 2001), proceso exacerbado por la necesidad de obtener recursos por parte de una población que se incrementa un $2,1 \%$ anual (FAO 2001) y que para el 2001 estaba en un $60 \%$ bajo la línea de pobreza en 6 de los 7 paises (PNUD 2003). En la Región imperan economias muy pequeñas, rezagadas y casi estancadas, un proceso intenso de apertura comercial, instituciones débiles y deficientes para ejercer un control adecuado de la tala y comercio ilegal de productos forestales. La distribución de los derechos de uso y propiedad de la tierra es desigual (Mendieta, 1993; Miller et al. 2001), con $80 \%$ de la tierra en manos de grandes terratenientes en el año 1976 (Mora-Escalante y Salas 1996, citado en UICN 2000).

Aún en medio de este panorama, se han experimentado importantes avances, particularmente durante los últimos 10 a 15 años. Aunque todavia en la década de los 80 no se tenian grandes logros sobre MFS (Poore et al,. 1989), hoy existen en la región 691.346 ha de bosques naturales y plantaciones forestales certificadas por el Forest Stewardship Council (FSC 2004), donde sobresalen las concesiones comunitarias del norte de Guatemala. Además, FAO (2001) estima que $13 \%$ del área boscosa se encuentra bajo planes de manejo y se ha establecido 462.000 ha de plantaciones forestales, principalmente en Costa Rica y Guatemala, donde se ha fomentado la actividad por medio de incentivos económicos (FAO, 2002). También se ha avanzado en la implementación de lineamientos y de criterios e indicadores de MFS, tanto en el ámbito regional, representado por el Proceso de Lepaterique, como en el nivel nacional (Louman et al, 2002).

También hay importantes cambios tendientes a la modernización y apertura de las instituciones públicas, acompañados de un proceso promisorio de integración regional (entre paises), del fortalecimiento de los gobiernos municipales (particularmente en Honduras y Nicaragua), asi como de las organizaciones del sector productivo privado (particularmente en Costa Rica y Guatemala) y de la sociedad civil en general. Lo anterior ha permitido una mayor participación en el diálogo político y en el manejo de los recursos forestales (por ejemplo, las mencionadas concesiones comunitarias), el desarrollo de instrumentos financieros que propician una valoración y pago por los servicios de los ecosistemas forestales. 
particularmente en Costa Rica (Campos et al., 2001) e iniciativas que promueven enfoques ecorregionales para el manejo sostenible de los recursos naturales, como el Corredor Biológico Mesoamericano (Miller, Chang and Johnson, 2001), que a la vez ha fomentado el establecimiento de corredores biológicos locales y binacionales, con ta-participación de gran diversidad de actores.

Es significativo el potencial de los mecanismos de pago por servicios ambientales (PSA) y de otros instrumentos de mercado para promover el EE en la región. EI PSA resulta entendible para la sociedad y para los tomadores de decisión pues se enfoca en los beneficios que esta recibe de los ecosistemas, además, si los responsables de manejar y conservar los recursos forestales perciben beneficios económicos netos por mantener el flujo de servicios para la sociedad, se facilita la implementación de prácticas sostenibles (Nasi et al., 2002; Rodriguez, 2002).

Grandes retos aun persisten, particularmente en cuanto a un procesamiento e industrialización más eficiente de la madera, mayor competitividad en la gestión especialmente de pequeñas y medianas empresas, acceso a mercados que recompensen un mejor desempeño ambiental y social, establecimiento de más plantaciones forestales y aumento del área de bosques naturales bajo MFS, particularmente en los países con mayor área de bosques, como Nicaragua y Honduras (Valle et al., 2001; Ortiz et al., 2002). Por otro lado, el conocimiento silvicultural de algunos ecosistemas forestales (i.e. manglares) es aun incipiente, mientras que su estado de conservación es muy pobre (Paniagua et al., 2001).

Adicionalmente, la Región cuenta actualmente con $22 \%$ de su extensión (13 millones de ha) como áreas protegidas, aunque falta mucho para consolidarlas y lograr sus objetivos de conservación, de beneficios para las poblaciones aledañas, e incorporar su manejo en estrategias integrales en escala de paisajes, tal como lo pretende el Corredor Biológico Mesoamericano, en coherencia con el equilibrio e integración entre uso y conservación que propone el EE.

Debido al alto grado de fragmentación de los paisajes forestales, al tamaño pequeño de las unidades de manejo y a la relevancia de las múltiples interrelaciones entre los componentes de estos paisajes (actores, usos de la tierra, escalas y disciplinas) (Sayer and Campbell, 2003), el MFS basado en el EE será de mayor provecho si se articula como parte de una gestión territorial a escala de paisaje, como por ejemplo las reservas de biosfera, cuencas hidrográficas, bosques modelo y corredores biológicos que se vienen implementando en la Región. El ordenamiento territorial debidamente planificado e implementado constituye un marco fundamental para la conservación y manejo sostenible de los bosques (Miller et al. 2001. PNUD 2003), según Vargas, (1992) $49 \%$ de la tierra centroamericana está sub- o sobreutilizada.

Por lo tanto, los principales desafios para el desarrollo del EE en relación con el MFS en América Central son de naturaleza social y económica, lo cual requiere de ajustes institucionales y en las politicas, para crear un entorno favorable al MFS, incluyendo el acceso a servicios técnicos y financieros para los productores forestales (particularmente los pequeños y medianos) y claridad en los derechos de propiedad y uso de estos recursos (Campos et al., 2004). 


\section{LOS ACTORES DEL MANEJO FORESTAL}

Los principales actores involucrados en la evolución del MFS centroamericano se agrupan en los sectores académico y científico, gubernamental, instituciones no gubernamentales, industria, organizaciones de productores y cooperación internacional. Para que el MFS sea aceptado y respetado por la sociedad como una estrategia de desarrollo, conforme al primer principio del EE, la historia centroamericana revela que estos actores necesitan condiciones e información mínimas que propicien un entorno favorable a este proceso (Nilson, 1999; Mora and Salas, 1996).

Probablemente el sector empresarial e industrial ha sido el último en manifestarse como tal en relación con el MFS, dado que este sector no tubo mayor consideración administrativa o técnica sobre el manejo adecuado durante la mayor parte de la historia. En Centroamérica la información sobre el significado del MFS se empezó a canalizar a fines de los años 80 a través de instituciones académicas, la cooperación internacional y algunas ONG, que ayudaron a generar conciencia sobre el tema y desarrollar de criterios técnicos adaptados a los ecosistemas locales.

Las escuelas universitarias de ingenieria forestal en Centroamérica se constituyeron en su mayoría en la década de los 70 , siendo en algunas regiones las primeras en establecer planes de manejo forestal y áreas demostrativas, con potencial de contribuir al balance usoconservación sugerido en el principio 10 del EE. A escala regional, el Centro Agronómico Tropical de Investigación y Enseñanza (CATIE) ha sido determinante en la investigación y desarrollo para el manejo sostenible de plantaciones forestales y bosques naturales, planteando desde un inicio un enfoque multifuncional de estos ecosistemas, dando énfasis al fortalecimiento del capital humano y la creación de operación de redes de cooperación horizontal entre los diversos actores en la región (Galloway, 2002), propia del espíritu de los principios 11 y 12 del EE.

Conforme el sector científico y académico internacional promovió los conceptos de MFS, estos se incorporaron en las academias locales, con influencia de la cooperación técnica internacional; desde ahi, estos conceptos empezaron a incidir sobre las políticas y legislación gubernamentales. Simultáneamente, los grupos ambientalistas han ejercido presión, llamando la atención por la degradación creciente de los ecosistemas forestales. Todo lo anterior ha redundado en una creciente reglamentación sobre el acceso y uso del recurso forestal, que aunada a su deterioro y escasez crecientes y a las incipientes tendencias de mercados más concientes, han provocado las primeras iniciativas de grupos empresariales por organizarse y asumir prácticas sostenibles de manejo. Paralelamente ha evolucionado la organización de pequeños productores forestales, la cual, sin embargo, sigue siendo aún insuficiente para avanzar hacia la sostenibilidad.

Algunas iniciativas relevantes de la cooperación internacional se enmarcan entre los llamados Integrated Conservation and Development Projetcs (ICDP); tales como el proyecto BOSCOSA (1989 - 1995) realizado en el sur de Costa Rica entre 1989 y 1995 por la Fundación Neotrópica, junto con WWF y USAID, y que planteó el MFS como herramienta para conservar la cobertura forestal y promover el desarrollo en la zona de amortiguamiento del Parque Nacional Corcovado (Donovan y Buschbacher 1989). Como otros ICDP, su experiencia reveló que el desarrollo rural es mucho más complejo y lento en su abordaje que el MFS, de modo que los resultados de mediano plazo, en atención a los principios 4 y 8 del EE, se 
optimizan al enfocar esfuerzos en el MFS, que a su vez requiere incentivos para hacerlo viable dentro del sistema productivo del campesino, de aqui surgió una de las primeras propuestas de sistema de pago por servicios ambientales (PSA) (Campos, 1992).

Otra enseñanza de los ICDP es la importancia de la organización y capacitación comunal en torno a un proceso productivo como el MFS, cuya relevancia sólo resulta visible en el mediano plazo, cuando la mayor parte de los proyectos ya han finalizado. Por otra parte, para que el MFS se consolide en las estrategias de vida de las comunidades, se requieren incentivos efectivos de corto, mediano y largo plazo, que ataquen las causas de la deforestación y degradación forestal. Han resultado además fundamentales la comunicación y colaboración entre proyectos, actores locales, regionales y nacionales, y la gestión participativa del MFS, contemplando su integración con diversos elementos del paisaje y un abordaje intersectorial e interdisciplinario.

El proyecto Conservación para el Desarrollo Sostenible en América Central, otro ejemplo de ICDP, ejecutado durante los años 90 por CATIE, junto con varios colaboradores locales y con financiamiento de los paises escandinavos, demostró la viabilidad de realizar MFS en bosques de menos de 50 ha, como parte integral de sistemas diversificados de finca agropecuaria, más aún cuando los finqueros hacen un procesamiento previo de la madera para venderla a mejores precios. Este proyecto encontró que para constituir en agentes de conservación del bosque a las comunidades del norte de Guatemala, estas debian contar con algunos servicios básicos fundamentales, una agricultura más sustentable y rentable, y con acceso legal al recurso forestal.

Dicho proyecto fue uno de los pioneros del proceso de concesiones forestales comunitarias, mediante las cuales los grupos comunales logran acceso legal y organizado al bosque, el cual protegen y aprovechan, de manera muchas veces más eficaz que el Estado en las áreas protegidas; lo que a la vez les ha permitido mejorar sus capacidades de organización, gestión empresarial, silvicultura y conciencia ambiental (Monroy, 2001; Reyes, Ammour, 1997; Jiménez, Reyes; 2001; CATIE, CONAP; 2001), a la vez que promueven su desarrollo socioeconómico (fuentes de empleo e ingresos, infraestructura comunal, mayor capacidad técnica, organizativa y administrativa, cambio de la percepción de los beneficios del bosque) (Carrera et al., 2002).

En procesos como el que se vive en el norte de Guatemala, el MFS se consolida cada vez más como una opción defendida y realizada por las comunidades, en cuyas organizaciones el Estado ha delegado la administración del bosque, los actores visualizan la estrecha interdependencia entre los sistemas agrícolas y forestales, la necesidad de que ambos sean rentables y por lo tanto bien administrados en términos ecológicos y financieros, y los campesinos asumen la conservación de la biodiversidad, constituyéndose en una de las manifestaciones más maduras de un EE (Carrera et al., 2002; Carrera et al., 2001).

El proyecto Cooperación en los Sectores Forestal y Maderero en Costa Rica (COSEFORMA) ejecutado por la GTZ alemana, el Ministerio del Ambiente y Energía, la Cámara Costarricense Forestal y el Instituto Tecnológico de Costa Rica, promovió la eficiencia industrial en la producción y el procesamiento, el desarrollo de mercados y la certificación para productos forestales. En Honduras, el Proyecto Desarrollo del Bosque Latifoliado (PDBL), financiado por la Agencia Canadiense para el Desarrollo Internacional, realizó una importante capacitación y formación de recursos humanos en aspectos de manejo forestal en el litoral Atlántico. 
Entre iniciativas locales innovadoras de los últimos años, la Fundación para el Desarrollo de la Cordillera Volcánica Central, procura una retribución equitativa a los productores con MFS en el norte y centro de Costa Rica, conforme al principio 4 del EE. Para ello ha promovido conceptos como la compra de madera por adelantado, donde el productor percibe un ingreso previo a la cosecha que contribuye a subsanar el problema de flujo de caja, y la certificación grupal de pequeños productores, quienes reciben asistencia técnica y logistica en aspectos de certificación, MFS y el acceso a incentivos como el PSA (Campos et al. 2004).

Estas iniciativas permiten constituir una nueva figura de propietario forestal, que valora al bosque como sistema productivo, a menudo en complemento con otros sistemas agropecuarios en la conformación de fincas más sostenibles (Miller et al. 2001, Repetto y Gillis 1998, Sandoval 2000). El papel de las ONG, con el aporte de la cooperación internacional, evoluciona asi desde estrategias paternalistas o que idealizaban de manera irreal las capacidades empresariales de los grupos comunitarios, hasta modelos que promueven las capacidades técnicas y empresariales de los usufructuarios del bosque, sean estos grupos comunitarios, pequeños propietarios o incluso medianos o grandes empresarios.

Surgen además nuevos conceptos de ONG, fruto de la iniciativa privada de los mismos propietarios del bosque, tal es el caso de la Asociación Costarricense de Reservas Privadas, que representa los intereses de 120 propietarios privados que voluntariamente conservan cerca de 60.000 ha de bosques naturales, alrededor de $50 \%$ de ellos para ofertar servicios a la creciente actividad turistica. Esta Red ha servido como base para iniciar la Red Centroamericana de Reservas Naturales Privadas (Sandi, 2003).

Los grupos "ambientalistas" también han jugado un rol fundamental, llamando constantemente la atención sobre las deficiencias en los sistemas de control y administración del recurso forestal, y actuando como fiscales permanentes de las iniciativas que inciden sobre el estado de los bosques. Tal fue el caso de las presiones ejercidas para evitar que la empresa Ston Forestal estableciera una planta de astillado de pulpa de madera de sus plantaciones en el Sur de Costa Rica, alegando que podría tener un gran impacto ecológico en el Golfo Dulce. Otras luchas abordadas por estos grupos resultan polémicas, como por ejemplo la que resultó en la eliminación del PSA para bosques bajo MFS en Costa Rica y que priva de este incentivo a quienes conservan los bosques no por medio de su protección absoluta sino del MFS; este bien puede ser un paso atrás, con respecto a la valoración multifuncional de los bosques (Campos et al., 2004).

Uno de los elementos más relevantes en la evolución reciente del MFS lo constituye la organización de los pequeños productores, para gestionar una legislación más favorable a sus necesidades a través del diálogo politico y para apoyarse mutuamente en el mejoramiento de sus capacidades empresariales. En tal contexto deben mencionarse organizaciones locales como la Junta Nacional Forestal Campesina (JUNAFORCA) en Costa Rica, que ha promovido una visión integradora del bosque primario y secundario como elemento de la finca agricola, o la Asociación de Comunidades Forestales de EI Petén (ACOFOP), que representa a 22 comunidades de esa zona guatemalteca y contribuye a su fortalecimiento en el proceso de las concesiones forestales.

En la escala regional, tiene particular relevancia política la Asociación Coordinadora Indigena y Campesina de Agroforesteria Comunitaria Centroamericana (ACICAFOC), 
organización de base que desde 1994 busca facilitar el acceso a servicios técnicos y financieros de estos sectores e "impulsa el ecodesarrollo y el empoderamiento de las comunidades, ... basado en sus experiencias, como respuesta práctica a la vulnerabilidad socio-ambiental y cultural de la región"1.

Los productores también se han organizado para mejorar técnicas de producción, procesamiento y comercialización, como por ejemplo en las concesiones forestales comunitarias de Guatemala, en la Cooperativa Regional Agroforestal Colón Atlántida Honduras Limitada en la región caribeña de ese pais, algunos grupos indigenas en La Mosquitia Hondureña, y la Comisión para el Desarrollo Forestal de San Carlos en Costa Rica, organización regional que representa más de 500 asociados. Muchos de estos grupos, sin embargo, aun no están consolidados, y fortalecer la organización de pequeños y medianos productores forestales es aun un tema prioritario, particularmente ante la reducción predominante en los servicios del Estado.

\section{CAMBIOS CLAVE EN LA MENTALIDAD}

La raiz de muchos obstáculos para un manejo forestal más sostenible y coherente con el EE en la Región es de carácter cultural histórico, dado que los modelos de desarrollo promovidos durante la historia post-colonial, hasta hace poco no consideraron al bosque como un sistema productivo, sino más bien como un obstáculo para el uso de las tierras con fines agricolas o urbanos. Con excepción de unas pocas especies de maderas consideradas preciosas, la mayor parte del recurso maderero fue quemado, en aras del cambio de uso de tierras forestales a agricolas. Hubo incentivos estatales para este cambio tales como "derechos de uso o de propiedad" a los cuales accedian quienes eliminaban áreas de bosque pristino, como parte de las politicas de colonización que predominaron hasta al menos la mitad del siglo XX. Tal sub-valoración, aunada a la abundancia aparente de la madera durante la mayor parte de la historia, favoreció sus bajos precios. Esta situación está cambiando, particularmente en las áreas más deforestadas, como Costa Rica, donde la escasez de las especies más valiosas ha permitido el ingreso de mayor diversidad de especies al mercado lo cual a su vez mejora la viabilidad del MFS y reduce la presión sobre muchas especies tradicionales cuyas poblaciones están amenazadas.

Por lo tanto, es probable que el cambio fundamental, con el cual se inicia la constitución de un entorno más favorable al MFS, es la reducción de incentivos históricos al cambio de uso de tierras forestales a agricolas, y la percepción creciente, por parte del estado y de la sociedad en general, del bosque como un sistema que produce bienes y servicios relevantes. EI MFS es aủn un concepto nuevo, que no ha tenido tiempo suficiente para difundirse y ser comprendido ni por parte de muchos propietarios del bosque, ni por la mayoria de la sociedad, un concepto con cerca de quince años de estarse promoviendo en los foros científicos, académicos y políticos, en contraposición a una cultura de culto a la deforestación de 500 años (Campos et al., 2002).

Este proceso avanza paulatinamente y con algunos tropiezos; como ejemplo de ello, aunque en la Costa Rica actual está prohibido el cambio de uso de áreas forestales, aún

http://www.acicafoc.net/home.php, consultado el 30 de junio 2004. 
generan polémica disposiciones politicas que permiten algún grado de cambio para infraestructura turística, y algunos propietarios de bosque usan ardides para disimular su eliminación paulatina. Este tipo de comportamiento conlleva, además de los elementos culturales descritos, la percepción de que el bosque es aun un tipo de uso de la tierra poco competitivo o rentable y a la vez se convierte en una competencia desleal contra aquellos que ofrecen productos de fuentes legales. Tal menosprecio es particularmente fuerte en relación con el bosque secundario, pese a que diversos estudios demuestran su potencial productivo (Finegan, 1992).

Por lo tanto, un reto pendiente es la competitividad del sector forestal, afectada ya sea porque en algunas regiones el mercado de maderas aun no se diversifica lo suficiente, por el bajo precio de las maderas (que tradicionalmente no considera el costo del buen manejo), o porque muchas iniciativas forestales comerciales adolecen de poca capacidad técnica para el MFS, ineficiencia empresarial y cadenas productivas inadecuadas. Por otra parte, los mercados evolucionan de manera dinámica y los productores deben organizarse y actualizarse en torno a estrategias acordes con condiciones como los tratados de libre comercio. A tono con los principios 4 y 9 del EE, en el futuro solo será viable un MFS en manos de productores prósperos, bien capacitados y con capacidad de gestión empresarial; no de campesinos pobres en condiciones de subsistencia.

Aunque se avanza en el desarrollo de capacidades técnicas y empresariales, aun los productores forestales afrontan altos costos de transacción, agravados por trámites complejos y excesivos. Se ha evolucionado desde políticas que fomentaban la deforestación hacia otras que pretenden proteger el bosque, pero que mal aplicadas o reglamentadas propician la corrupción y las actividades ilegales de extracción, transporte y comercio de madera. Es menester aprovechar toda la experiencia acumulada y la tecnologia existente para desarrollar mecanismos de control de bajo costo (para el estado y para los productores), eficientes y que no den ventaja a quienes promueven la ilegalidad.

En este sentido se destacan las politicas tendientes a descentralizar el control de la actividad forestal, tales como el sistema de regentes forestales de Costa Rica, que son técnicos privados que gozan de fe pública para el monitoreo técnico del MFS, o el Fondo de Manejo Forestal en Honduras. También las iniciativas para una mayor participación de las comunidades locales rurales en la administración del recurso forestal estatal, donde sobresalen las concesiones forestales comunitarias en el norte de Guatemala (Carrera et al., 2002). Otras estrategias que amplian la participación de los actores de diversos ámbitos incluyen la conformación de redes de cooperación horizontal del sector forestal en Honduras y Nicaragua.

Una iniciativa actual interesante, afín al principio 4 del EE, es el Programa de Competitividad de las Pequeñas y Medianas Empresas Forestales ${ }^{2}$, realizado por CATIE con apoyo del BID en Guatemala, Honduras y Nicaragua, dirigido a desarrollar actitudes empresariales de los miembros que componen los eslabones de la cadena forestal. Se actualiza a los socios con información comercial de productos estrella, para motivar a

Alejandro Santos, Comunicación personal.

2005. Lider Programa de Competitividad de las Pequeñas y Medianas Empresas Forestales en América Central.

CATIE-IDB. Managua 
abastecedores y procesadores a emprender un negocio conjunto, y experimentar como cadena productiva las responsabilidades que implica elaborar un producto en particular, de manera sostenible.

Ha sido difícil que los grandes avances en los conceptos técnicos de manejo se vean reflejados en las prácticas realizadas en el campo. Es por eso que los procesos para desarrollar estándares con principios, criterios e indicadores (C\&l) constituyen un insumo valioso para orientar y monitorear las técnicas de manejo de manera práctica (McGinley, Finegan, 2002). En tal sentido se han dado esfuerzos de carácter regional, como el proceso de C\&I de Lepaterique, y avances locales como el de los C\&I de MFS que deben cumplirse por ley para la aprobación de los planes de manejo en Costa Rica (Comisión Nacional de Certificación Forestal, 1999).

El ámbito de incentivos a la actividad forestal revela ejemplos de evolución y aprendizaje propios de un manejo adaptativo De modelos poco eficaces, como los primeros incentivos a la reforestación en Costa Rica, que en algunos casos fueron captados por empresarios cuyo interés principal fue la sustitución del pago de impuestos y que no contaron con un seguimiento técnico adecuado, se ha pasado a sistemas de incentivos más equitativos y exitosos. Tal es el caso de los administrados por el Instituto Nacional de Bosques en Guatemala; o del innovador sistema de Pago por Servicios Ambientales (PSA) en Costa Rica (Rojas, Aylward, 2003; Rodriguez, 2002), establecido por la Ley Forestal de 1996, a cargo del Fondo Nacional de Financiamiento Forestal (FONAFIFO), una institución descentralizada que administra fondos de un impuesto a los combustibles fósiles y de negociaciones internacionales de venta de servicios ambientales (GEF, Banco Mundial, Alemania y Noruega). Este PSA se basa en los principios de quien contamina debe pagar por ese impacto, quien disfruta de los servicios debe pagar por los mismos y quien provee estos servicios conservando el bosque debe recibir un pago justo por ellos.

EI PSA en Costa Rica reconoce los servicios de mitigación de gases de efecto invernadero, protección de fuentes de agua, de biodiversidad y ecosistemas y de su belleza escénica. Su éxito hace que los recursos no logran atender la demanda existente. Paralelamente, en el pais existen iniciativas privadas de PSA por parte de empresas que requieren garantizar la conservación de fuentes de agua con diversos fines (Cordero y Castro, 2001; Nasi et al., 2002; Rodriguez, 2002). Para el año 2003, FONAFIFO ${ }^{3}$ contrató para el PSA 63.329 ha de bosques bajo protección, 3.254 ha para reforestación y 325 ha de plantaciones establecidas, para un total de 66.911 ha. Desde su inicio en 1997 hasta lo que va del 2004, el PSA ha incorporado a mas de 400.000 ha en manos de más de 6.000 productores, con una inversión cercana a los US\$100 millones.

Aunque todavia son pocos, debe destacarse el aporte a la consolidación de un sector forestal más sostenible que brindan las empresas forestales de mayor escala o poder económico que asumen prácticas de MFS. Se puede citar como ejemplos Precious Woods en Costa Rica y Nicaragua, que fomenta la reforestación con altos estándares ambientales, integrando las plantaciones en el paisaje con áreas de vegetación natural; el Grupo Nova

${ }_{3}^{3}$ FONAFIFO en linea, agosto 2004: http://www.fonafifo.com 
con un enfoque similar para la reforestación en Panamá (ECOFOREST), Guatemala y Costa Rica; y la empresa Pórtico, que exporta puertas elaboradas con maderas de bosques naturales de la costa Atlántica de Costa Rica y Nicaragua y que se venden en mercados que exigen la certificación del FSC.

La certificación forestal es un elemento fundamental que genera expectativas de un mercado que retribuya los esfuerzos por la conservación de los ecosistemas forestales, aunque el mismo aún no se consolida (Louman et al., 2002). La cantidad de unidades de manejo certificadas es creciente, y para ello ha sido fundamental la asistencia técnica y la facilitación de trámites brindada por algunas ONG's locales, como por ejemplo Naturaleza para la Vida en Guatemala o FUNDECOR en Costa Rica, o internacionales, como el Fondo Mundial para la Naturaleza (WWF), que cuenta con una oficina para Centroamérica. Carrera et al. (2004) analizan en detalle el estado y evolución de la certificación forestal en Guatemala.

\section{LOS ESLABONES DE LA CADENA PRODUCTIVA}

En contraposición a una historia centroamericana, en la cual el bosque ha sido visto básicamente como un obstáculo para el desarrollo, la lucha actual por la conservación del recurso forestal se centra en definir y promover el desarrollo de cadenas productivas eficaces, que retribuyan el esfuerzo que conlleva el MFS, complementado además por el dificil y a veces infructuoso esfuerzo de los estados por conservar áreas protegidas (Louman, 2003).

Los eslabones de la cadena productiva de los productos forestales posteriores a la extracción de la materia prima desde el bosque, son los que menos atención han recibido por parte de las instituciones del sector (Louman, 2003). La mayor evolución de las técnicas para la elaboración de los productos finales se ha dado fuera de la Región, lo cual atenta contra la consolidación de una cultura forestal, pues la materia prima que sale del bosque se vende muy barata, sin representar una alternativa económica estable para quien hace usufructo de la tierra, mientras que en los procesos de aserrio y desarrollo de productos finales predomina la ineficiencia y la escasez de ejemplos donde se procure un alto valor agregado. En este contexto el aprovechamiento del bosque aparece vinculado a la pobreza de la mayoria de los actores involucrados y a la riqueza de pocos de ellos, en contraposición a los principios de equidad del EE.

El personal encargado del procesamiento rara vez cuenta con una capacitación formal sobre su trabajo, lo que fomenta la ineficiencia en el uso y mantenimiento de los equipos. Estos equipos, a su vez, suelen ser obsoletos y en muchas ocasiones adquiridos a partir de errores en los ámbitos ejecutivos de las instituciones y las empresas, que instalan industrias cuya capacidad no es consistente con la oferta sostenible del bosque. La falta de capacitación de operarios no solo se refleja no solo en las etapas de procesamiento, sino en diversas etapas del aprovechamiento forestal y la falta de un sistema que acredite y valore con un salario adecuado a los técnicos. Esta situación refleja la falta de estrategias integrales de formación de los recursos humanos.

La integración entre los paises tiene importantes implicaciones, Centroamérica es una unidad fisiográfica con elementos en común y de tamaño relativamente pequeño, de manera tal que conforme a los principios 3 y 7 del EE, resulta relevante el análisis regional 
de las interacciones y los impactos de las acciones sobre los ecosistemas. Las organizaciones de carácter regional juegan un papel clave para promover esta visión, tal es el caso del proyecto Corredor Biológico Mesoamericano (CBM) o el de la Comisión Centroamericana de Ambiente y Desarrollo (CCAD) ${ }^{4}$. Actualmente, son crecientes las iniciativas de planificación ecoregional, que procuran un análisis integral a mayores escalas. Por otra parte los mercados locales son pequeños y muy sensibles a situaciones globales como la importación de madera de otras regiones, la cual se ha incrementado al entrar en vigencia nuevos tratados comerciales. Las estrategias regionales de mercadeo podrían contribuir a la gestión de mercados más estables o a la consolidación de empresas locales que den valor agregado a los productos.

Actualmente, a la luz de una visión más madura de la cadena productiva, se busca que el mercado reconozca la inversión adicional que conlleva el MFS, por medio de la certificación forestal. En el norte de Costa Rica se ha comprobado científicamente la contribución de la certificación a mejorar las prácticas silviculturales, aunque aún los mercados para productos certificados están poco desarrollados (Campos et al., 2004).

El afán por la conservación de los bosques ha provocado que se enfaticen las medidas encaminadas al control excesivo de las actividades de manejo, a tal grado que muchas veces resulta más atractivo para los actores de la cadena productiva buscar diversas formas de aprovechamiento y comercialización ilegal antes que llenar todos los requisitos legales que imponen las autoridades estatales. Uno de los principales retos actuales es lograr un balance entre el control efectivo y la simplificación de trámites en torno a la cadena. La lucha contra la ilegalidad debe entenderse como un proceso más integral y complejo que el control policial o el que se pretende a través de los trámites burocráticos. Las autoridades están llamadas a entender las raices culturales y económicas de las actividades ilegales. análisis que debe ser compartido con los principales actores sociales, como los grupos ambientalistas que con frecuencia tienden a promover las medidas de restricción, sin darse cuenta que su efecto final puede ser negativo para los bosques, y para su posibilidad de constituirse en la base de sistemas productivos y de equidad, conforme al EE (Campos et al. 2002).

\section{LOS MOTORES DEL CAMBIO}

Antes de los años 90, ni el manejo forestal ni la protección absoluta de los bosques pudieron desacelerar la deforestación de los bosques en América Central, cuyas tasas regionales tuvieron un promedio de $2,1 \%$ anual entre 1980 y 1990 (FAO, 1997). La experiencia acumulada llevó a cuestionar las estrategias de manejo y de protección utilizadas y la polarización entre ambas, y a analizar los problemas de fondo de la deforestación y degradación de los bosques. Pese a ello, para el período entre 1990 y 2000 (FAO 2001) la deforestación regional fue todavia alta $(1,6 \%)$. Quizás, el cambio más significativo ha sido la aceptación de la integración entre desarrollo y conservación, que permite un mayor énfasis en los aspectos humanos en las actividades y politicas orientadas al MFS, y contribuye con otros cambios, como la descentralización del sector forestal, la cooperación regional y la

\footnotetext{
${ }^{4}$ http://www.ccad.ws/antecedentes/antecedenteshistoricos.htm, consultado el 30 de junio 2004
} 
conciencia de la necesidad de monitorear los impactos ecológicos y socioeconómicos de las actividades realizadas (Carrera et al., 2001; McGinley, Finegan, 2002).

A continuación se discute algunos factores relevantes que facilitaron este proceso, muchos relacionados con cambios en las relaciones entre la gente y los recursos naturales, entre los grupos de actores, la tecnologia y el conocimiento que apoya estas relaciones. Se agrupan en relación con la percepción del bosque por la sociedad, las relaciones entre la gente y el bosque, y las relaciones entre grupos de interés.

\section{Una Revolución Cultural: la Percepción del Bosque}

El primer principio del EE presenta el manejo de los recursos como un asunto de decisión de los actores sociales. En tal marco resulta fundamental que ante la sociedad centroamericana la conservación del bosque deje de considerarse un esfuerzo inútil o de beneficios ajenos a muchos ciudadanos. Además de las mejoras en los contenidos curriculares y en el acceso a la educación de la población, algunos elementos de crisis han contribuido a una mayor valoración social del bosque en los últimos años, tales como la relación que de manera creciente establecen los ciudadanos entre la conservación del bosque y el abastecimiento de agua o la prevención de los efectos de desastres naturales producto de sequias, lluvias o huracanes.

De particular influencia sobre la percepción de la población centroamericana fue el Huracán Mitch, que en 1998 ocasionó enormes pérdidas humanas y pérdidas materiales valoradas en US \$ 6 mil millones (Kandel, Rosa, 2000). Los cuestionamientos subsecuentes sobre la relación entre el manejo del paisaje y la susceptibilidad a las lluvias, fueron seguidos de iniciativas hacia un manejo integrado de las cuencas hidrográficas, que ahora incluyen conceptos como el co-manejo adaptativo. Sin embargo, también han surgido elementos a favor de un desempeño más competitivo del bosque como sistema productivo, tanto relativos a condiciones para el aprovechamiento y comercio de la madera, como a la diversificación de los bienes y servicios aprovechados. Una nueva alternativa que crea un escenario favorable al desarrollo de MFS, lo constituyen las expectativas de mercados de carbono dentro del Clean Development Mechanism, particularmente en el ámbito de las plantaciones forestales y los sistemas agroforestales (Campos et al. 2000).

La diversidad de productos del bosque no es ajena a algunas sociedades rurales, particularmente las indígenas, pero no son tantos los productos en la región que acceden a mercados formales. La Plataforma de Yucatán, que comprende regiones de México, Belice y Guatemala, tiene una gran tradición de aprovechamiento de productos forestales no maderables (PFNM) para el mercado internacional. Con la institucionalización de las concesiones forestales comunitarias, se crean condiciones más propicias para el manejo sostenible de las poblaciones de estas especies (Carrera et al., 2002; Mollinedo et al., 2002).

Un elemento novedoso es la experiencia del Instituto Nacional de Biodiversidad de Costa Rica (INBio), una ONG que desde 1989 ha negociado contratos de bioprospección por más de US $\$ 2.5$ millones con grandes empresas o instituciones de países desarrollados. 
Parte de este dinero lo recibe el Estado para el manejo de los parques nacionales donde se realiza la prospección (Laird, 2002). Además, los contratos indican que en caso de que algún principio químico inspirado u obtenido de la biodiversidad local resulte la base de una actividad comercial, un porcentaje de las ganancias obtenidas será para el INBio y para el Sistema Nacional de Áreas de Conservación. Esta iniciativa, interesante y polémica, constituye una experiencia en el contexto del balance entre conservación y el uso promovida por el EE.

La actividad turística se ha convertido en uno de los principales alicientes para la conservación del bosque en tierras no estatales y en uno de los servicios más atractivos que brinda el bosque para hacer económicamente rentable su conservación. Algunas experiencias hacen de la ecología y del aprovechamiento forestal sostenible, parte de los atractivos turísticos mostrados (Otárola, 2001). Los turistas son muchas veces estudiantes, a veces en visita de gira técnica. Se evoluciona hacia un turismo que retribuye al propietario del bosque su conservación y que puede aportar más beneficios a los habitantes rurales, conforme al espiritu de equidad del EE.

\section{Las Relaciones entre la Gente y los Recursos Naturales}

Entre los factores importantes que inciden en las relaciones entre la gente y los recursos naturales se encuentran el aumento en la fragmentación de los bosques centroamericanos (Miller et al., 2001), la mayor proporción de la población con problemas de acceso a agua potable y los cada vez mayores impactos resultantes de fenómenos naturales (particularmente huracanes, tormentas tropicales y sequias).

Son preocupantes las tasas de pobreza en las zonas rurales y, aunque la situación mejoró un poco durante la última década del siglo $\mathrm{XX}$, ello se tradujo en más personas con un empleo remunerado, pero en varios países con un menor salario promedio (PNUD, 2003) y un aumentó de la brecha entre ricos y pobres. Tal situación acompaña a la deforestación y fragmentación, que reduce la disponibilidad de los bienes y servicios forestales para la población; además resulta en el uso inadecuado de la tierra, reduciendo aún más las oportunidades económicas de los pobres.

Aunque en general el uso de productos forestales es adicional al de los productos agropecuarios, en los países más pobres de la Región, como Nicaragua, hasta el $68 \%$ de la población total depende por lo menos parcialmente de leña para poder consumir estos productos (PNUD, 2003). Además, muchas familias generan ingresos extras al involucrarse en el aprovechamiento de la madera, sobre todo en áreas alejadas de los centros poblacionales, donde también ocurren la mayoria de las actividades ilícitas. Aunque en el corto plazo, las actividades agropecuarias parecen ofrecer mayores oportunidades económicas para estas familias, a menudo no son sostenibles o son muy vulnerables a fenómenos climáticos y a los mercados, mientras se están perdiendo las oportunidades ofrecidas por los ecosistemas forestales en el mediano y largo plazo.

Uno de los principales problemas es que las familias rurales no perciben una retribución por los beneficios de los bosques. Durante la última década, muchos esfuerzos se han dirigido 
a aumentar el valor de los bosques para sus dueños y para las comunidades vecinas. Existen estudios sobre el potencial del aprovechamiento de PFNM del bosque primario (Godoy et al., 2000; Mollinedo et al., 2002; Villalobos, 2003) y secundario (Santana et al., 2002). Sin embargo, probablemente su mayor valor está en los servicios ambientales, que según estimaciones del Banco Mundial pueden significar un $72 \%$ del valor económico total de los bosques (Camino et al., 2002).

El sistema de pago por servicios ambientales (PSA) en Costa Rica, antes descrito, está orientado a transferir parte de los costos de la manutención de las funciones y servicios forestales a los beneficiarios finales de estos. Este sistema considerado modelo, sin embargo, se ha criticado por no contribuir a mejorar la conservación de áreas criticas ni las estrategias de vida de los productores pequeños, quienes suelen estar en condiciones legales. económicas y de acceso a información que les dificultan acceder a estos incentivos (Nasi et al., 2002). Debido a ello se realizan ajustes en el programa y se fomentan investigaciones sobre el valor de los servicios e identificación de áreas prioritarias para la conservación, como corredores biológicos y cuencas.

Por otra parte, existen estudios que indican que el buen manejo forestal, según estándares reconocidos en el ámbito internacional, no necesariamente afecta negativamente la biodiversidad (Aguilar et al., 2000; Delgado et al., 1997; Ordóñez, 2003), lo que puede ser aún mejorado con la aplicación de mecanismos de apoyo al buen manejo, como el PSA y la certificación (Campos et al., 2004) confirmando asi su potencial cómo actividad económica amigable con el ambiente (Finegan et al., 1993) y su papel potencial en la conservación y el desarrollo rural.

Las fuerzas de mercado y los efectos de la remoción de una gran parte de la vegetación boscosa sobre el régimen local de agua también han sido catalizadores para la adaptación de medidas a favor del manejo en escala de paisaje y una mayor participación local en este manejo (Camino, 1993), procesos que a menudo contribuyen a mejorar las capacidades organizativas para el desarrollo, a veces con apoyo de iniciativas institucionales positivas, como los centros agrícolas cantonales que operan en Costa Rica desde 1975 y han permitido enfocar los beneficios de programas nacionales hacia los pequeños productores rurales (Salazar, 2003).

Los análisis de los efectos del huracán Mitch (Kandel, Rosa, 2000) y otros estudios sobre las estrategias de vida de familias rurales han mostrado que los bosques contribuyen a mitigar las consecuencias de este tipo de fenómenos. Cuando las cosechas o el mercado fallan o los hogares son destruidos, el acceso a los bosques, aunque sean fragmentos o secundarios, puede ayudar a superar periodos críticos (Vásquez Vásquez, 1999). Por otra parte, los estudios indican que con la organización local, la planificación del uso de la tierra y el cumplimiento de las necesidades inmediatas, particularmente de los grupos más pobres, se fortalece la capacidad de reducir y enfrentar las consecuencias de los desastres.

El Huracán Mitch, en particular, llevó a los gobiernos locales y la cooperación internacional a repensar las estrategias de desarrollo. Mientras los paises buscaron sobre todo mejores oportunidades económicas en la arena internacional, la cooperación enfatizó 
"la vulnerabilidad social y ecológica, la transparencia, participación y el desarrollo local" (Kandel, Rosa, 2000). Los desastres naturales como el Mitch han funcionado como catalizadores de procesos ya en marcha. El interés actual por implementar más acciones en torno al manejo integrado de cuencas, creará nuevas oportunidades de un desarrollo forestal acorde al EE.

El efecto de la deforestación y la fragmentación sobre la diversidad biológica se percibe como menos urgente, ya que actualmente afecta menos al desarrollo socioeconómico de los habitantes de la Región. Sin embargo, se estima que ya existen más de 50 especies de fauna y 200 especies de plantas en peligro de extinción en toda la Región (PNUD, 2003). En algunos paises se ha adaptado legislación forestal y ambiental para lograr la conservación de estas especies. Un caso bien estudiado en Costa Rica es la relación entre la lapa verde (Ara ambigua) y el árbol almendro de montaña (Dipteryx panamensis) donde anida y se alimenta, y que en 1996 condujo a la restricción del aprovechamiento del árbol a sólo $50 \%$ de los individuos entre 70 y $120 \mathrm{~cm}$ de DAP. A pesar de esta restricción, la distribución y cantidad de lapas observadas se ha reducido, probablemente por la tala y la fragmentación del bosque (Chassot et al. 2001, Ramos 2004). Se evidencia la necesidad de una planificación en escala de paisaje, con participación de los dueños de terrenos, de instancias relevantes y de grupos con intereses especificos (turismo, conservación, agricultores) para lograr la conservación efectiva de especies en peligro. Estos estudios han contribuido a impulsar la participación local y la formación del Parque Nacional Maquenque en Costa Rica y el corredor biológico binacional Rio San Juan - La Selva.

\section{Las Relaciones entre Diferentes Grupos de Interés}

La formalización de la participación de comunidades en el manejo forestal es otro cambio relevante de las últimas décadas. En 1974 se inició el Sistema Social Forestal en Honduras, posteriormente fortalecido en el norte del país con el apoyo de la cooperación internacional y la formación de Áreas de Manejo Integral (AMI) (Mendieta 1993), lográndose casos de buen manejo como alternativa económicamente viable y de contención al avance de la frontera agricola (Caballero et al., 2002). En el área central de Honduras se inició en 1992 el proyecto MAFOR, que fomentó el manejo de bosques de pino por comunidades y pequeños propietarios, que involucró a los dueños en el manejo y procesamiento por medio de planes sencillos y del fomento de empresas pequeñas de aserrio (Lazo, 2001 y Scherr 2000 citados en Scherr et al. 2004).

Las concesiones forestales comunitarias en Guatemala, integran procesos de conservación y desarrollo, que además de reconocer los derechos de uso de los diferentes actores, incorporan un sistema de control (monitoreo estatal en combinación con la certificación forestal), agregan valor al producto forestal, aumentan los conocimientos técnicos de los actores y su información tanto sobre los recursos como sobre el manejo de los mismos. Además, las tecnologias de sensores remotos facilitaron la planificación y monitoreo espacial, fortaleciendo los procesos de MFS por actores de pocos recursos y sus ONG acompañantes (Carrera et al., 2002). 
Esfuerzos como los mencionados, y otras iniciativas de grupos comunitarios e indigenas en la Región, son apoyados por ACICAFOC (antes descrita), cuyos grupos miembros, completamente autónomos, tienen actividades de manejo y uso forestal que abarcan mas de 2 millones de hectáreas, y que gracias a la asociación participan de intercambios entre organizaciones, para comunicar lecciones aprendidas en el proceso de negociación entre las comunidades y diferentes instancias de gobierno (Chinchilla, 2002; UICN, 2000). En los años 90 crecieron otras iniciativas de comunicación entre actores, como las redes operativas de cooperación horizontal (Galloway 2001, 2002), que buscan una planificación estratégica participativa del manejo de los recursos naturales locales en el norte de Honduras y sur de Nicaragua.

En el ámbito regional hubo avances en la integración entre paises, reflejada en la creación de la Comisión Centroamericana del Ambiente y Desarrollo (CCAD), como responsable oficial ministerial para dar seguimiento a los acuerdos de Rio 1992. CCAD ha sido protagonista principal en la firma de la Alianza para el Desarrollo Sostenible (ALIDES) en 1994, el impulso de proyectos regionales de conservación (sistema mesoamericano de áreas protegidas y Corredor Biológico Mesoamericano), el Plan de Acción Forestal Centroamericano que dio paso a la Estrategia Forestal Centroamericana, el proceso de criterios e indicadores para el manejo de Lepaterique y el fortalecimiento de los marcos legales y politicos de los paises centroamericanos.

\section{ALGUNAS CONCLUSIONES PARA ORIENTAR EL MFS HACIA EL EE}

Para comprender la evolución de los ecosistemas forestales en Centroamérica se debe colocar en el centro de los análisis a la gente, su cultura, historia, características y necesidades.

Es necesario desarrollar mecanismos financieros apropiados para el MFS, que reconozcan las externalidades del MFS y las condiciones de los diversos actores.

Es necesario entender la economia de la corrupción, sus motivaciones y raices, de manera que las politicas incentiven el MFS y lo hagan más competitivo.

Es necesario cambiar de un enfoque de subvención a un enfoque de inversión, particularmente en áreas rurales.

Se debe poner más énfasis en el manejo integrado del paisaje, entender y administrar los bosques en su interacción con los diversos componentes, actores y sectores del paisaje.

Tal enfoque debe reflejarse en la educación, particularmente de posgrado, para contar con técnicos capaces en temas especificos, pero también profesionales experimentados formados en el manejo integrado de los recursos naturales con una visión sistémica de los territorios. 
Es necesario crear conocimientos y educar personas para crear condiciones y capacidades para la participación y la distribución equitativa de los beneficios de la conservación.

La investigación es clave, pero debe ser relevante y traducirse en herramientas y decisiones sencillas y prácticas, tanto en el nivel político como en el de las prácticas de manejo.

La sostenibilidad ecológica es un proceso continuo y probablemente infinito de aprendizaje, pero

se sabe suficiente para hacer un buen manejo forestal bajo un enfoque adaptativo.

Debe ser constante la implementación y actualización de mecanismos para el monitoreo de las actividades de MFS, la caracterización del impacto y la definición de ajustes necesarios.

Tal monitoreo no se debe limitar al bosque que constituye una UMF, sino a todo el paisaje, en el marco de regiones biofísica, ecológica o politicamente funcionales.

\section{REFERENCIAS}

Aguilar-Amuchastegui, N., Finegan, B., Louman, B.; Delgado, D., 2000. Patrones de Respuesta de Scarabaeinae a las Actividades de Manejo en Bosques Naturales Tropicales. Revista Forestal Centroamericana 30:40-45.

Caballero, M., Cardona, L., Sánchez, A. Isidro, C., 2002. Manejo Forestal con Participación Comunitaria en la Costa Norte de Honduras. In: Sabogal, C., Silva, J.N.M. (Eds), Aplicando Resultados de Pesquisa, Envolvendo Atores e Definindo Políticas Públicas. Embrapa Amazonia Oriental y Ministerio de Agricultura, Pecuaria e Abastecimiento, Belém, Brasil. Pp.199-207.

Camacho, M., Finegan, B., 1997. Efectos del Aprovechamiento Forestal y el Tratamiento Silvicultural en un Bosque Húmedo del Noreste de Costa Rica. El Crecimiento Diamétrico con Enfasis en el Rodal Comercial. Serie Técnica, Informe Técnico No. 295. CATIE, Turrialba, Costa Rica. 54pp.

Campos A, J.J., 1992. Incentives and Campesino Participation in Forestry Development: the Case of the Osa Peninsula. Invited Paper at the Central American Workshop on Forest Incentives. World Wildlife Fund (WWF) and the Nicaraguan Forestry Action Plan. Managua, Nicaragua. August, 26-27, 1992.

Campos A., J.J, Ortiz, R., Smith, J., Maldonado, T., Camino, T. de, 2000. Almacenamiento de Carbono y Conservación de Biodiversidad por Medio de Actividades Forestales en el Área de Conservación Cordillera Volcánica Central, Costa Rica. Potencialidades y Limitantes. Serie Técnica, Informe Técnico No. 314. CATIE, Turrialba, Costa Rica. 70pp.

Campos A., J.J., Finegan, B. Villalobos, R., 2001. Management of Goods and Services from Neotropical Forests Biodiversity: Diversified Forest Management in Mesoamerica. In: Assessment, Conservation and Sustainable Use of Forest Biodiversity. CBD Technical Series No. 3. Secretariat of the Convention on Biological Diversity. Montreal. Pp. 5-16. 
Campos A., J.J., Camacho, M., Villalobos, R., Rodriguez, C.M. Gómez, M., 2002. Tala Ilegal en Costa Rica: Problemática y propuestas de solución. Biocenosis 16(1-2):40-46.

Campos A., J.J., Louman, B., Locatelli, B., Garay, M., Yalle, S., Villalobos, R., López, G., Carrera, F. (in press). Efectos del Pago por Servicios Ambientales y la Certificación Forestal en el Desempeño Ambiental y Socioeconómico del Manejo de Bosques Naturales en Costa Rica. Serie Técnica. Informe Técnico.

Camino, R. de, 1993. El Papel del Bosque Húmedo Tropical en el Desarrollo Sostenible de América Central: Desafios y Posibles Soluciones. Revista Forestal Centroamericana 6:7-16.

Carrera, F., Pinelo, G., 1995. Prácticas Mejoradas para Aprovechamientos Forestales de Bajo Impacto. Serie Oficial, Informe Técnico No. 262. CATIE, Turrialba. 60pp.

Carrera, J.R., Campos A., J.J., Morales, J. Louman, B., 2001. Evaluación de Indicadores para el Monitoreo de Concesiones Forestales en Petén, Guatemala. Revista Forestal Centroamericana 34:8488.

Carrera, F., Morales, J., Galvez, J., 2002. Concesiones Forestales Comunitarias en la Reserva Biosfera Maya, Petén, Guatemala. In: Sabogal, C., Silva, JNM. (Eds), Aplicando Resultados de Pesquisa, Envolvendo Atores e Definindo Politicas Públicas. Embrapa Amazonia Oriental I Ministerio de Agricultura, Pecuaria e Abastecimiento, Belém, Brazil. Pp193-198.

Carrera, F., Stoian, D., Campos A., J.J., Pinelo, G. Morales, J., (In press). Forest Certification in Guatemala. In: University of Yale (Ed.). Forest Certification in Developing Countries and Countries in Transition. Paper presented at the Symposium «Forest Certification in Developing Countries and Countries in Transition» held at the University of Yale on June 10-14, 2004.

CATIE, CONAP, 2001. Plan General de Manejo Forestal Diversificado de la Concesión Comunitaria de San Miguel La Palotada. Serie Técnica, Informe Técnico No. 320. CATIE, Turrialba, Costa Rica. 98pp.

Chassot, O., Monge, G., Powell, G., Palmintiri, S., Alemán, U., Wrighht, P., Adamek, K., 2001. Lapa Verde, Victima del Manejo Forestal Insostenible. Ciencias Ambientales 21:60-69.

Chassot, O., Monge, G., Ruiz, A. Mariscal, T., 2003 Corredor Biológico Costa Rica - Nicaragua Pro Lapa Verde. Ambientico No.114. On Line: http://www.una.ac.cr/ambi/Ambien-Tico/114/chassot.html.

Chinchilla, A., 2002. Central America: ACICAFOC, An On-going Proposal. WRM Bulletin no 63. Consultado en internet 30 junio 2004 en: http://www.wrm.org.uy/bulletin/63/Acicafoc.html

Clark, D.A., 1998. Deciphering Landscape Mosaics of Neotropical Trees: GIS and Systematic Sampling Provide New Views of Tropical Rainforest Diversity. Annals of the Missouri Botanical Garden 85(1):1833.

Comisión Nacional de Certificación Forestal, 1999. Estándares y Procedimientos para el Manejo Sostenible y la Certificación Forestal en Costa Rica. Comisión Nacional de Certificación Forestal. Costa Rica. 54 p.

Cordero, D., Castro, E., 2001. Pago por Servicio Ambiental Hidrico. Revista Forestal Centroamericana 36:41-45.

Delgado, D., Finegan, B., Zamora, N. Meir, P., 1997. Efectos del Aprovechamiento Forestal y el Tratamiento Silvicultural en un Bosque Húmedo del Noroeste de Costa Rica. Serie Técnica, Informe Técnico No. 298. CATIE, Turrialba, Costa Rica. 43pp. 
Donovan, R. Buschbacher, R., 1989. The Forest Conservation and Management Project (Boscosa) of the Osa Peninsula, Costa Rica. Annual Report and Work Plan. Fundación Neotrópica, World Wildlife Fund. 29pp.

FAO, 1997. State of the World's Forests 1997. Food and Agriculture Organization of the United Nations, Rome. 200pp.

FAO, 2001. State of the World's Forests 2001. Food and Agriculture Organization of the United Nations, Rome. $181 \mathrm{pp}$.

FAO, 2002. Evaluación de los Recursos Forestales Mundiales 2000. Informe Principal. Estudio FAO Montes 140. Organización de las Naciones Unidas para la Agricultura y la Alimentación. Roma. 468pp.

Finegan, B., 1992. El potencial de Manejo de los Bosques Húmedos Secundarios Neotropicales de las Tierras Bajas. Serie Técnica, Informe Técnico No. 188. CATIE, Tirrialba, Costa Rica. 29pp.

Finegan, B., Sabogal, C., Reiche, C. Hutchinson, I., 1993. Los Bosques Húmedos Tropicales de América Central: Su Manejo Sostenible es Posible y Rentable. Revista Forestal Centroamericana 6:1727.

Forest Stewardship Council, 2004. Forests Certified by FCS-Accredited Certification Bodies. Consulted June 23rd 2004. International Center. Bonn, Germany. www.fscoax.org

Galloway, G., 2001. Redes Operativas: Un Mecanismo Efectivo para Promocionar el Manejo de Bosques Tropicales. Revista Forestal Centroamericana 33:33-37.

Galloway, G., 2002. Las Redes Operativas y su Papel en la Política Forestal. Experiencias Prometedoras en Honduras y Nicaragua. Revista Forestal Centroamericana 37:26-32.

Godoy, R., Wilkie, D., Overman, H., Cubas, A., Cubas, G., Demmer, J., McSweeney, K. Brokaw, N., 2000. Valuation of Consumption and Sale of Forest Goods from a Central American Rain Forest. Nature 406(6791):62-63.

Jiménez, J. Reyes, R., 2001. Experiencias sobre la Introducción de Alternativas Productivas en una Concesión Forestal Comunitaria de Petén, Guatemala. Serie Técnica, Informe Técnico No. 316. CATIE, Turrialba, Costa Rica. 48pp.

Laird, S. (Ed), 2002. Biodiversity and Traditional Knowledge. Equitable Partnerships in Practice. Earthscan, London. 504 pp.

Kandel, S., Rosa, H., 2000. Después del Mitch: Temas y Actores en la Agenda de Transformación de Centroamérica. In: EIRD/OPS (Estrategia Internacional para la Reducción de Desastres /Organización Panamericana de la Salud) (compiladores). Huracán Mitch: Una Mirada a Algunas Tendencias Temáticas para la Reducción del Riesgo. EIRD, San José, Costa Rica. P.21-57.

Lazo, F., 2001. El Papel Forestal en la Mitigación de la Pobreza; Perfil de Honduras. Background Paper for Inter-agency Forum on "The Role of Forestry in Poverty Alleviation", Tuscany, Italy, September 2001. FAO, Rome.

Lopez, G., (in prep). Environmental and Scioeconomic Effects of the Payment of Environmental Services and Forest Certification of Natural Forest Management in Costa Rica. Draft Paper to be Submitted to Journal of Forest Policy and Economics. 
Louman, B., Campos A., J.J., Schmidt, S., Zagt, R. Haripersaud, P., 2002. Los Procesos Nacionales de Certificación Forestal y su Relación con la Investigación Forestal. Interacciones entre Politicas y Manejo Forestal, casos de Costa Rica y Guyana. Revista Forestal Centroamericana 37:41-46.

Louman, B., 2003. Cadena Forestal: Cuándo Funciona y por qué no Funciona siempre. In: Cadenas de Producción para el Desarrollo Económico Local y el Uso Sostenible de la Biodiversidad. Compendio del Seminario Internacional. Managua, 17 al 19 de marzo del 2003. Pp.95-100.

Louman, B., Garay, M., Yalle, S., Campos A., J.J., Locatelli, B., Villalobos, R., López, G.

Carrera, F., 2004. Environmental and Socioeconomic Effects of the Payment of Environmental Services and Forest Certification of Natural Forest Management in Costa Rica. Draft paper to be submitted to Journal of Forest Policy and Economics.

McGinley, K. Finegan, B., 2002. Evaluations for Sustainable Forest Management. Serie Técnica, Informe Técnico No. 238. CATIE, Turrialba, Costa Rica. 73 .

Mendietta, M., 1993. Manejo Sustentable del Bosque Húmedo Tropical en Honduras: Experiencias de la Región Forestal Atlántida. Revista Forestal Centroamericana 6:28-37.

Miller, K., Chang, E., Johnson, N. 2001. En Busca de un Enfoque Común para el Corredor Biológico Mesoamericano. World Resources Institute.Washignton DC, USA. 49pp.

Mollinedo, A., Campos A., J.J., Kanninen, M. Gómez, M., 2002. Beneficios Sociales y Rentabilidad Financiera del Manejo Forestal Ccomunitario en la Reserva de la Biosfera Maya, Guatemala. Serie Técnica, Informe Técnico No. 327 CATIE, Turrialba, Costa Rica. 39pp.

Monroy, H., 2001. Manual de Planificación y Ejecución de Aprovechamientos Forestales en las Concesiones Comunitarias de Petén. Serie Técnica, Manual Técnico No. 47. CATIE, Turrialba, Costa Rica. 84pp.

Mora-Escalante, J. Salas, A., 1996. Contexto Regional para el Uso Sostenible de los Recursos Naturales en América Central (documento para la discusión). Unión Mundial para la Naturaleza. Oficina Regional para Mesoamérica (UICN-HORMA). 20pp.

Nasi, R., Wunder, S. Campos A., J.J., 2002. Forest Ecosystem Services: Can They Pay our Way Out of Deforestation? Discussion Pper Pesented for the Frestry Roundtable of GEF and UNFF II, Costa Rica, March 11, 2002. New York. 29 pages plus annexes.

Nilsson, M., 1999. Conceptos Básicos en el Trabajo con Bosques y Comunidades. Serie Técnica, Boletin Técnico No. 307. CATIE, Turrialba, Costa Rica. 46pp.

Ordoñez, Y., 2003. Validación de Indicadores Eológicos para la Evaluación de Sostenibilidad en Bosques Bajo Manejo Forestal en el Trópico Húmedo, con Enfasis en Bosques de Alto Valor para la Conservación. Tesis Mag. Sc. CATIE, Turrialba, Costa Rica.

Ortiz, S., Carrera, F., Ormeño, L.M., 2002. Comercialización de Productos Maderables en Concesiones Forestales Comunitarias en Petén, Guatemala. Serie Tècnica, Informe Técnico No. 326. CATIE, Turrialba, Costa Rica. 39pp.

Otárola, M., 2001. Análisis de Preferencias para la Evaluación de la Compatibilidad de Actividades Turisticas y Silvicolas en Robledales de la Parte Alta y Media de la Cuenca del Rio Grande de Orosi, Costa Rica. Tesis Mag. Sc. CATIE, Turrialba, Costa Rica. 89pp. 
Paniagua, C.; Cajina O.; Marmillod, D., 2001. Primer Caso de Manejo Forestal Comunitario en Manglares de Nicaragua: Experiencias de la cooperativa 28 de julio. Serie Técnica, Informe Técnico No. 318. CATIE, Turrialba, Costa Rica. 102pp.

Poore, D., 1989. Conclusions. In: Poore, D., Burgess, P., Palmer, J., Rietbergen, S., Synnott, T. (Eds) 1989. No Timber Without Trees: Sustainability in the Tropical Forest, Earthscan, London. pp 190-209.

Poore, D. (Ed.), 1989. No Timber without Trees. Sustainability in the Tropical Forest. Earthscan, London. 252pp.

PNUD, 2003. Segundo Informe sobre Desarrollo Humano en Centroamérica y Panamá. San Josè. Costa Rica. Disponible en http://www.estadonacion.or.cr, consultado el 20 de junio 2004.

Ramos, Z., 2004. Estructura y Composición de un Paisaje Boscoso Fragmentado: Herramienta para el Diseño de Estrategias de Conservación de la Biodiversidad. Thesis Mag. Sc. CATIE, Turrialba, Costa Rica.

Repetto, R., Gillis, M., 1988. Public Policies and the Misuse of Forest Resources. Cambridge University Press, Cambridge, 211pp.

Reyes, R.; Ammour, T., 1997. Sostenibilidad de los Sistemas de Producción en la Concesión Comunitaria de San Miguel, Petén, Guatemala. Serie Petén No. 1. CATIE, Turrialba, Costa Rica. 30pp.

Rodriguez, J., 2002. Los Servicios Ambientales del Bosque: El Ejemplo de Costa Rica. Revista Forestal Centroamericana 37:47-53.

Rojas, M., Aylward, B., 2003. What Are We Learning from Experiences with Markets for Environmental Services in Costa Rica? A Review and Critique of the Literature. International Institute for Environment and Development. London. 102pp.

Salazar, M., 2003. Evaluación de la Restauración del Paisaje en el Cantón de Hojancha, Costa Rica. Thesis Mag. Sc.. CATIE, Turrialba, Costa Rica. 98pp.

Sandoval, R., 2000. Honduras: Su Gente, su Tierra y su Bosque. Tomo I. Graficentro editores. Tegucigalpa, Honduras. 476pp.

Santana, R., Montagnini, F., Louman, B., Villalobos, R. Gómez, M., 2002. Productos de Bosques Secundarios del Sur de Nicaragua con Potencial para la Elaboración de Artesanias de Masaya. Revista Forestal Centroamericana 38:85-90.

Sayer, J.A. Campbell, B., 2003. Research to Integrate Productivity Enhancement, Environmental Protection, and Human Development. In: Campbell, B., Sayer, J.A. Integrated Natural Resources Management. Linking Productivity, The Environment and Development. CABI Publishing. UK. Pp.1-15.

Sabogal, C., Castillo, A., Carrera, F. Castañeda, A., 2001. Aprovechamiento Forestal Mejorado en Bosques de Producción. Serie Técnica, Informe Técnico No. 323. CATIE, Turrialba, Costa Rica. 57pp.

Sabogal, C., Castillo, A., Mejia, A. Castañeda, A., 2001. Aplicación de un Tratamiento Silvicultural Experimental en un Bosque de La Lupe, Rio San Juan, Nicaragua. Serie Técnica, Informe Técnico No. 324. CATIE, Turrialba, Costa Rica. 37pp.

Sandi, C., 2003. Conservación Privada en Centroamérica. Ambientico No. 120. On Line: http:// www.una.ac.cr/ambi/Ambien-Tico/120/Sandi.htm 
Scherr, J.S., 2000. A Downward Spiral? Research Evidence on the Relationship Between Poverty and Natural Resource Degradation. Food Policy 25:479-498.

Scherr, J.S., White, A. Kaimowitz, D., 2004. A New Agenda for Forest Conservation and Poverty Reduction: Making Markets Work for Low-income Producers. Forest Trends/CIFOR/IUCN, Washington DC. $160 p p$.

UICN, 2000. Comunidad y Gestión de Bosques en Mesoamérica. Perfil Regional del Grupo de Trabajo sobre Participación Comunitaria en el Manejo de los Bosques. CICAFOC/UNOFOC/IUCN, San José, Costa Rica. 117pp.

Valle, L.F., Cruz, A. Centeno, G.A., 200 . Estado Actual del Manejo Forestal en Honduras. Consultores Forestales de Honduras FORESTA. Proyecto Información y Análisis para el Manejo Forestal Sostenible. Santiago de Chile.

Vargas, U.G., 1992. Estudio de Uso Actual y Capacidad de Uso de la Tierra en América Central. Anuario de Estudios Centroamericanos 18, vol 2.

Vásquez, A.A., 1999. Determinación de la Contribución del Bosque Secundario a la Economia de Familias Rurales de la Zona Norte y Sur de Honduras. Thesis Mag. Sc. CATIE, Turrialba, Costa Rica. $122 \mathrm{pp}$.

Villalobos, R.; Ocampo, R. (eds.), 1997. Productos No Maderables del Bosque en Centroamérica y El Caribe. Actas de la Consulta sobre la Situación de los Productos Forestales No Madereros, 17 al 21 de julio de 1995. Turrialba, Costa Rica, CATIE. Serie Técnica, Eventos Especiales No. 1. 103 p.

Villalobos, R., 2003. El Comercio de Productos No Maderables: Estímulo o Escollo para la Promoción del Manejo Forestal Sostenible. In. Cadenas de Producción para el Desarrollo Económico Local y el Uso Sostenible de la Biodiversidad. Compendio del Seminario Internacional. Managua, 17 al 19 de marzo del 2003. p 26-28. 